\title{
Localization of a Target with Three Degrees of Freedom Using a Low Cost Wireless Infrared Sensor Network
}

\author{
Nikos PETRELLIS, Fotios GIOULEKAS, Michael BIRBAS, John KIKIDIS \\ Analogies S.A., Patras Science Park, Platani-Rio, Patras, Greece \\ Email: \{nikos.petrellis, fotios.gioulekas, michael.birbas, john.kikidis\}@analogies.eu \\ Received August 10, 2009; revised September 14, 2009; accepted September 15, 2009
}

\begin{abstract}
The estimation of the position of a mobile target on a plane as well as its orientation is an important aspect for many applications. The indoor or outdoor localization of such a target has been widely addressed in the literature but if a third degree of freedom like rotation has to be also taken into consideration the difficulty in estimating the target position and orientation is significantly increased. A network consisting of only a small number of low cost infrared transmitters/receivers is used in this paper to estimate the position of a mobile target on a plane as well as its draft orientation with an angular step of $45^{\circ}$ or less. The distance and orientation estimation is based on the success rate that infrared patterns are retrieved at the target. This success rate parameter is calculated by simple ultra low cost microcontrollers. The architectural complexity and cost of the overall localization system is significantly lower than other approaches without sacrificing speed and accuracy. An error correction scheme like Turbo decoding is applied in order to increase the reliability and stability of the results by correcting burst errors introduced by real time noise.
\end{abstract}

Keywords: Position Estimation, Localization, Infrared Sensor Networks, Turbo Codes

\section{Introduction}

The applications where indoor localization is important concern robotics, automation, virtual reality and pervasive computing environments. Although knowing the position of a moving target on a plane is very important it would be valuable for a large number of applications if the orientation of the target could also be estimated. For example, a system guiding a robot, a handicapped person or a person wandering in a virtual museum, should also be aware of the draft at least orientation of this robot or person. Although several approaches have been proposed for the position estimation of targets with 2 degrees of freedom, there are not many general solutions for targets with 3 degrees of freedom.

A rather complicated method used by the robots in order to familiarize with an unknown environment is based on processing the images captured by cameras that are mounted on the robots in order to recognize landmarks and their distance [1]. Conclusions extracted from image processing are combined with other localization approaches [2] in autonomous robotics applications. Stochastic processing can also help in this case for the validation of an estimated position. It is obvious that image processing requires powerful and complicated processing units leading to rather expensive localization solutions.
Measuring the time of the flight of a reflected impulse wave or the strength of a signal are ordinary methods used for the indoor or even outdoor localization. Optical or Laser beams can be used to scan the surrounding area detecting the distance of walls or other obstacles. The cost of this solution is higher since very short time intervals have to be measured with high precision $[1,3]$. Ultrasonic signals can offer a lower cost alternative to this approach since the sonar waves travel with much lower speed than light $[4,5]$. A drawback concerning the use of ultrasounds is that this type of signal is not directional enough and it is difficult to isolate the sonar transmitter from the receiver in order to ensure that only the reflected signal will be taken into consideration during the distance measurement. Localization systems based on ultrasonic waves often estimate distances by measuring phase shifts of the original and the reflected signal, which also requires processing of high precision and speed.

The signal strength of multiple transmitters surrounding the target can also provide an indication about the target position using a triangulation method [6,7]. This technique has already been adopted in cellular telephony, Wireless Local Access Network (WLAN) or Bluetooth applications.

Magnetic fields have been adopted for the accurate non-contact control of the position and orientation of 
tools and medical instruments in short distances (up to a few centimeters) [8,9]; Distance estimation of up to $10 \mathrm{~m}$ using magnetic fields has been reported in [10].

Passive infrared sensors are used by mobile targets in order to avoid obstacles while active infrared tranceivers are employed similarly with laser or ultrasonic beams to detect the distance of the target from walls, obstacles etc $[11,12]$. Another interesting use of infrared light is the profiling of the surface of an object by recognizing its texture (used for distinguishing metal from plastic surfaces etc) $[13,14]$.

Low cost infrared tranceivers have been used in order to estimate the position of a moving target with 2 degrees of freedom with an absolute error of less than $20 \mathrm{~cm}$ in [15-18]. The reception quality of the digital patterns that are sent by at least two transmitters that are placed around the covered area is exploited for the estimation of the position of a receiver that can move on a plane without rotating. A calibration procedure before real time operation is required in order to familiarize the target with the area. During this calibration stage, the target visits predetermined positions and enumerates the recognized patterns in a period of time in order to estimate the success rate of the reception. A position identity can be formed by the success rates of the various pattern types that are employed. During real time operation, a position identity is constructed in the same way for the current target position and is compared to the identities that were estimated during the calibration stage. The closer position that was visited during the calibration is selected. A regression technique can help reaching a more accurate estimation of the real target position.

Instant noise that has not been taken into consideration during the calibration stage can reduce the speed and accuracy of the position estimation. Several rules can be applied to validate the results of the position estimation procedure [16]. If a positive estimation is characterized by these rules as unacceptable, it is discarded and a new estimation is initiated. Error Correction techniques have also been employed by the authors [17] in order to reduce the effect of instant noise and speed up the estimation procedure. The interleaving process employed in Turbo decoding can minimize the effect of the burst errors caused by the instant noise $[19,20]$. When the attenuated signal is received at the target, it is corrected by a decoder that can be implemented either in software or by dedicated hardware [21,22]. Since our localization approach is based on the quality of the received signal, the intension is to minimize the effect of the burst errors caused by instant noise through the selected Error Correcting method rather than fully recover the initial patterns. A description of how an additional sensor at the side of the target can be used to provide an orientation indication has been given in [18].

In the present work we modify some architectural features of the infrared transmitters and receivers in order to obtain a draft indication of the target orientation in $45^{\circ}$ angular steps i.e, 8 potential directions. It will also be explained how narrower angular steps and consequently more accurate orientation estimation can been supported. The position and orientation estimation in the architecture that will be presented here, takes place in two phases: the draft orientation is initially estimated by classifying the retrieved success rates as strong or weak and then the target position coordinates related to an infrared transmitter are estimated using the exact success rate values.

The designed localization system covers a large area with a small number of low cost transmitters/receivers since it is based on a simple digital processing method that merely counts patterns. A very good trade off between cost, area covered, accuracy and speed is achieved.

The architecture of the infrared transmitting and receiving devices along with the estimation methods used in our previous approaches are described in Section 2. The topology used in the present work and the position/orientation estimation method is described in Section 3. Finally, the experimental results will be discussed in Section 4.

\section{System Architecture}

\subsection{Infrared Transmitter (Irtx)}

The architecture of the infrared transmitters (IRTX) that are used in the proposed localization system is presented in Figure 1. A processing unit generates both the patterns and the carrier that are mixed and amplified before they are transmitted by one or more infrared emitting diodes. In $[15,16,18]$ the infrared patterns transmitted by an IRTX device had the form that appears in Figure 2a. Each pattern type consists of a number (i) of pulses that have a duration inversely proportional to the number $i$.

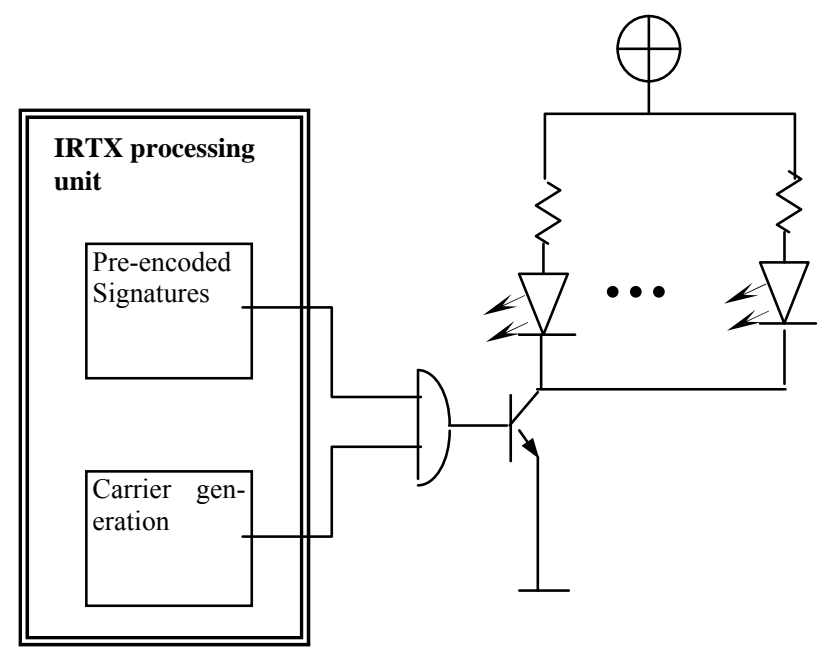

Figure 1. Architecture of an IRTX device. 




(a)

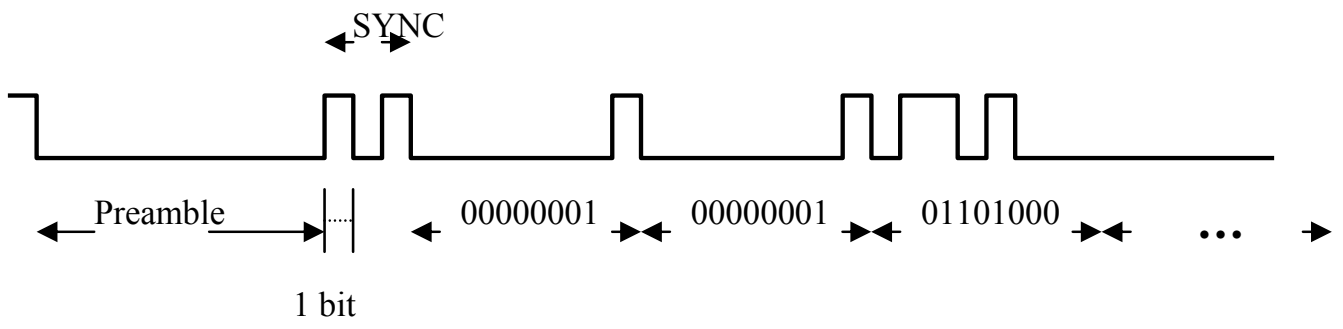

(b)

Figure 2. Infrared pattern formats consisting of a preamble followed by several MODi patterns (a) or signatures (b).
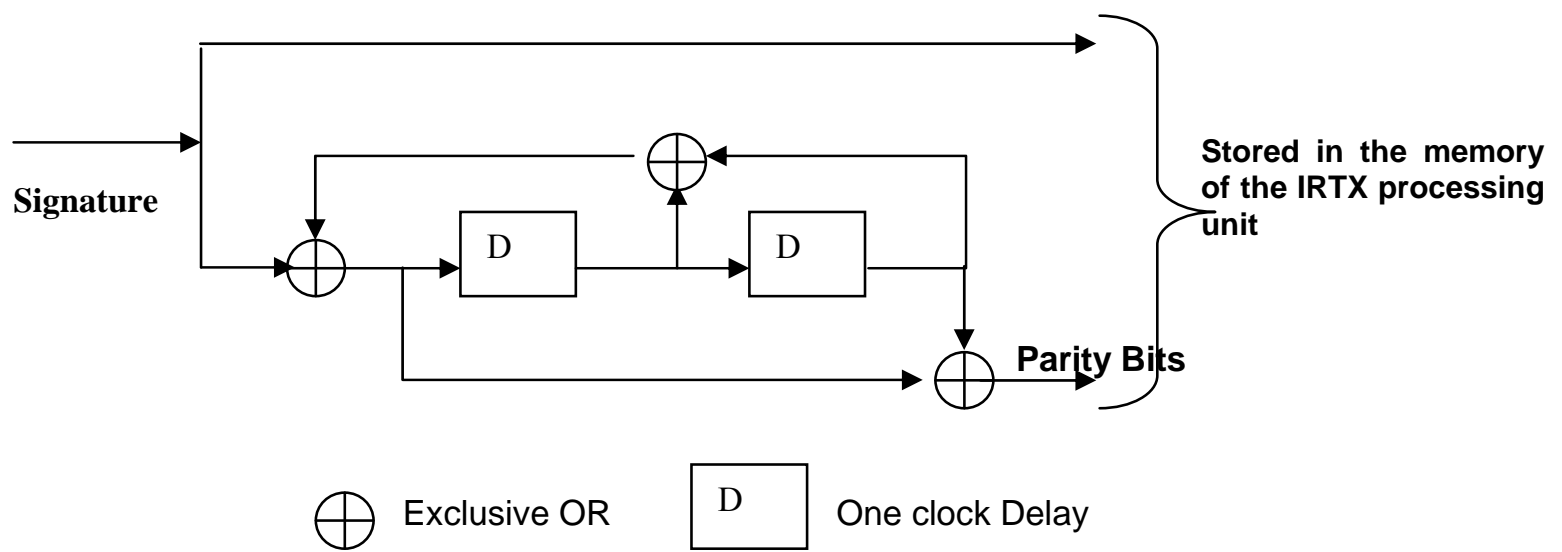

D One clock Delay

Figure 3. Recursive systematic convolutional (RSC) encoding used by the IRTX devices.

Such a pattern is designated as MODi. This type of format was chosen because patterns with higher number of short pulses are recognized with lower success rate than patterns consisting of lower number of long pulses. Moreover, the receiver may simply count rising or falling edges between long pause intervals in order to recognize a pattern type. Each IRTX device initially transmits a preamble and then, a specific number of codes from each pattern type. After the transmission of all the supported pattern types, this procedure is repeated by transmitting a new preamble.

The digital pattern signal is mixed with a carrier before driving the infrared emitting diodes. The carrier is used in order to avoid interference from other infrared sources like sunlight. More than one infrared emitting diodes can be connected in parallel and placed in a circular arrangement in order to cover a wider area.

Using a pattern format that consists of a constant number of bits that have equal duration as shown in Figure $2 \mathrm{~b}$ in order to employ an error correcting method at the re- ceiver can lead to faster implementations with higher precision results as discussed in [17]. Using this type of patterns, the receiver sensors have to take samples at regular time intervals instead of merely counting rising or falling edges. A preamble is initially transmitted followed by a synchronization sequence (SYNC). The preamble is a long pause period while the SYNC is a small sequence of identical pulses used by the receiver for synchronization. Then, all the supported pattern codes are transmitted. Patterns of this type will be called henceforth "signatures" since they identify their transmitter. Although multiple signatures with smaller length can be used, a single signature with 160-bit length can lead to a better trade off between speed and accuracy as discussed in [17].

The signatures are transmitted along with their parity bits that have been generated through a proper encoding. The receiver is aware of the supported patterns or signatures and simply enumerates the recognized ones i.e., those that have not been distorted. For this reason, pre- 
encoded signatures and parity bits are stored in the memory of the IRTX processing unit. The Recursive Systematic Convolutional (RSC) encoding described by Figure 3 is used off line to generate the parity bits that correspond to the supported signatures. The polynomial describing this encoding is:

$$
\left(1+\mathrm{D}^{2}\right) /\left(1+\mathrm{D}+\mathrm{D}^{2}\right)
$$

\subsection{Infrared Receiver (IRRX)}

The architecture of the infrared receiver devices (IRRX) used, is shown in Figure 4. The infrared sensors are connected to a bandpass filter to select only the infrared signals modulated at the specific carrier frequency used. Then, an integrator rejects the carrier and the pure pattern signal is recognized by the processing unit of the receiver. If the target moves with 2 degrees of freedom, two infrared sensors $\left(\operatorname{IRRX}_{\mathrm{A}}\right.$ and $\left.\mathrm{IRRX}_{\mathrm{B}}\right)$ are adequate [15-17]. If the target is also allowed to rotate a third IRRX sensor may be helpful as discussed in [18]. Nevertheless, in the next section we will describe how only 2 IRRX sensors can also be used in order to get an indication of the orientation of the target if they are placed at a proper angle.

The IRRX processing unit marks the start and the end of the signature and parity bit parts. The next stage is the application of a Turbo Decoding algorithm in order to minimize the effect of the burst errors caused by instant noise that has not been taken into consideration during the calibration stage [17]. It is noticed that the aim of the applied Turbo decoding method is to smooth the error occurrence instead of correcting all the errors. More specifically, the interleaving used by the Turbo decoders, scatters the burst errors throughout the length of a signature making easier their correction. The turbo decoder can be implemented at the host computer where the application that exploits the position estimation results is running. If the IRRX processing unit is a more complicated one supporting all the applications required by the target, the Turbo decoding can be implemented in a low level language like assembly. Another option is to embed a special Turbo decoding peripheral like the one described in [21]. This option has a higher cost but would significantly speed up the decoding procedure.

\subsection{Turbo Decoding Implementation Alternatives}

The state machine and the Trellis diagram that correspond to the encoding scheme of Figure 3 are presented in Figures $5 \mathrm{a}$ and $5 \mathrm{~b}$ respectively. The interleaver used is of the random type and it is shown in Figure 6 since a lower Bit Error Rate (BER) can be achieved in this way [17].

The Turbo Decoder used consists of two Soft In Soft Out (SISO) decoders each one of them operating alternatively on the un-interleaved and interleaved input signatures respectively (see Figure 7). Each SISO decoder consists of identical blocks that implement a stage of the Trellis diagram (Figure 8). A SISO decoder generates extrinsic information that is used as intrinsic input by the other SISO decoder at the following iteration. The decoding stops and the outputs are settled after a specific number of iterations.

The parameters $a \_S(t)$ and $b \_S(t)$ of Fig. 8 are branch metrics of the Trellis diagram qualifying how possible is a path leading to state $S$ of the stage $t$. $\operatorname{Int}(t), \operatorname{Ext}(t)$ and $U(t)$ are the intrinsic, the extrinsic info and the output of this stage. The inputs $\mathrm{P}(\mathrm{Yu} \mid \mathrm{Xu})$ and $\mathrm{P}(\mathrm{Yp} \mid \mathrm{Xp})$ are the

\section{Host Computer}

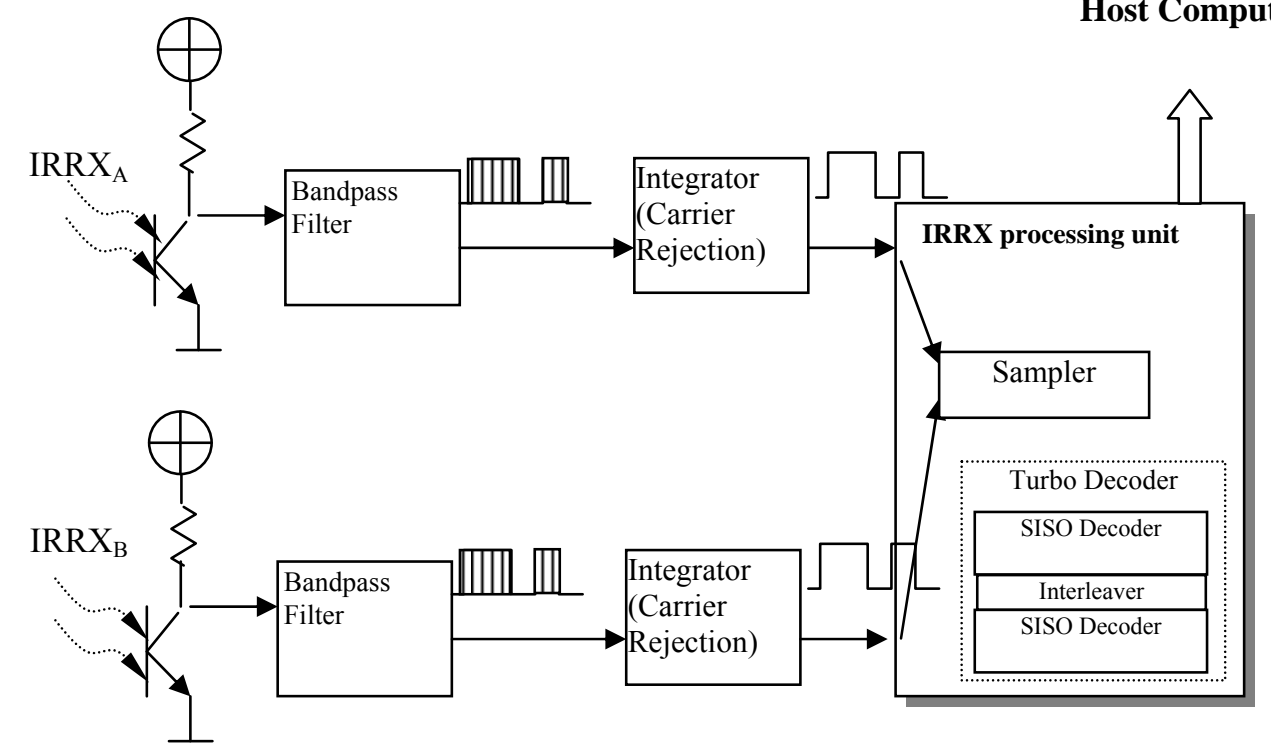

Figure 4. Architecture of the IRRX device consisting of two sensors and a processing unit that communicates with a Host Computer. 


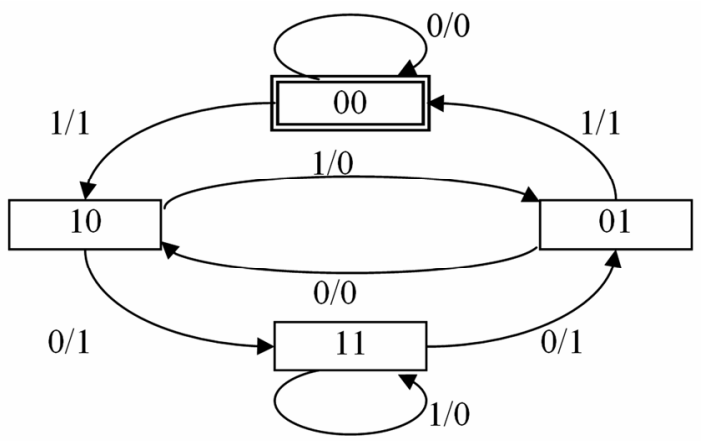

(a)

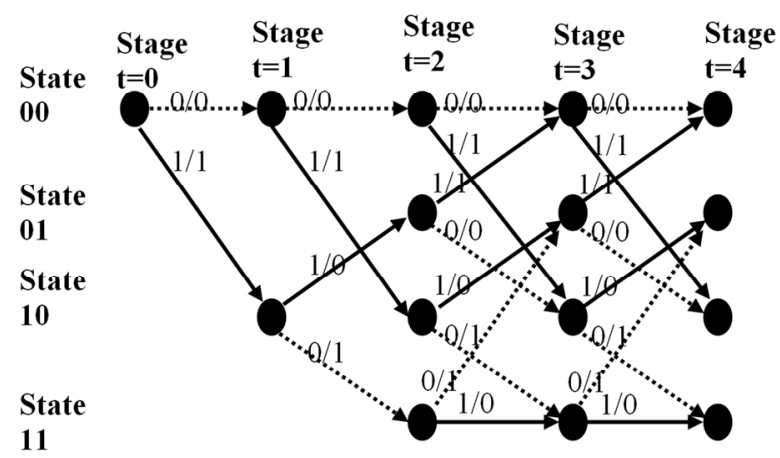

(b)

Figure 5. State machine (a) and Trellis diagram (b) for the RSC encoder of Figure 3.

160-bit Signature

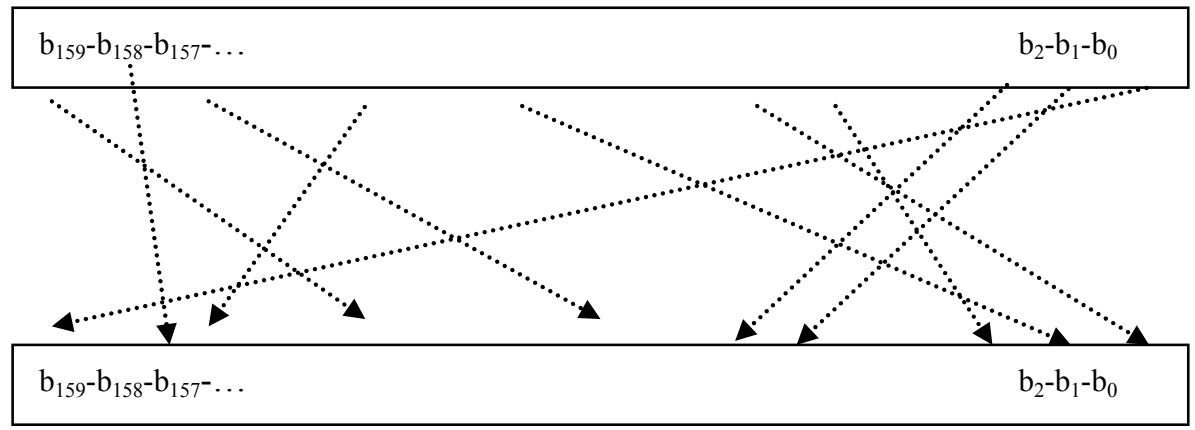

Figure 6. The bit positions of the original signature are mapped to random positions in the interleaved one (random interleaver).

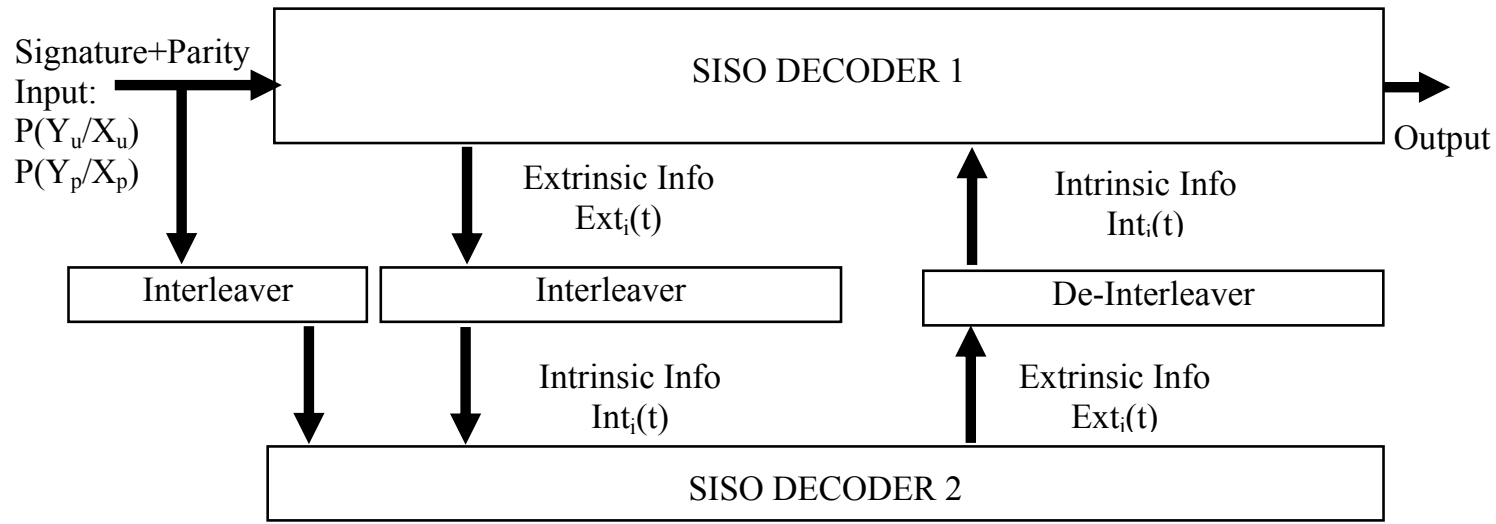

Figure 7. Turbo decoding scheme.

probabilities to receive symbols $\mathrm{Yu}$ and $\mathrm{Yp}$ given that the input $\mathrm{Xu}$ and the parity $\mathrm{Xp}$ has been transmitted. The lower BER is achieved if the decoding is performed by the Sum-Product Algorithm (SPA) that is described by the relations of Table 1 .

The analog implementation of a Turbo Decoder presented in [21] is an example of how the decoding needed by the localization system can be implemented as a processing unit peripheral. If simpler operations than multiplication are needed in order to have a less complicated hardware implementation of the Turbo decoding, the Max-Log MAP or the Min-Sum algorithm can be employed [22]. Their relations are derived by the equations of Table 1 if a logarithmic operation is applied to 




Figure 8. Implementation of a Trellis stage.

Table 1. The relations of the SPA algorithm.

\begin{tabular}{|c|c|}
\hline Block & Calculation \\
\hline A & $\begin{array}{c}\gamma_{i j}^{\prime}=\operatorname{Pr}\left(y_{u}(t) \mid x_{u}(t)=i\right) \operatorname{Pr}\left(y_{p}(t) \mid x_{p}(t)=j\right), \\
i, j \in\{0,1\}\end{array}$ \\
\hline B & $\gamma_{i j}=\gamma_{i j}^{\prime} \operatorname{Int}_{i}(t), \quad i, j \in\{0,1\}$ \\
\hline $\mathrm{C}$ & $a_{-} S(t)=\sum_{S^{\prime}} a_{-} S^{\prime}(t-1) \gamma_{S^{\prime} \rightarrow S}$ \\
\hline D & $b_{-} S^{\prime}(t)=\sum_{S} b_{-} S(t+1) \gamma_{S^{\prime} \rightarrow S}$ \\
\hline E & $I_{i j}=\sum_{i / j, S^{\prime} \rightarrow S} a_{-} S^{\prime}(t-1) b_{-} S(t)$ \\
\hline $\mathrm{F}$ & $\operatorname{Ext}_{i}(t)=\sum_{j} I_{i j} \operatorname{Pr}\left(y_{p}(t) \mid x_{p}(t)=j\right), i \in\{0,1\}$ \\
\hline G & $U_{i}(t)=\sum_{j} I_{i j} \gamma_{i j}, i \in\{0,1\}$ \\
\hline
\end{tabular}

both sides and the following identity is taken into consideration.

$$
-\ln \left(e^{-x}+e^{-y}\right)=\min (x, y)-\ln \left(1+e^{-|y-x|}\right)
$$

The second term of the right side of Equation (2) is often neglected with a penalty of about $0.5 \mathrm{~dB}$ in the achieved BER. In the present work the Turbo decoding scheme is implemented by software at the Host Computer. For this reason, SPA algorithm was chosen in order to achieve the best possible error correction.

\subsection{Topologies for Position Estimation}

In [15] the success rates of various pattern types at regular distance and angular steps was measured as shown in
Figure 9. The position of the target was represented with polar coordinates related to an IRTX device. For example, Figure 10 shows the success rate curves of pattern types MOD2, MOD5, MOD6 and MOD9 at 2m distance and angular displacement ranging from $-90^{\circ}$ to $+90^{\circ}$. If the target resides at a distance different than $2 \mathrm{~m}$, the success rates that are measured for MOD2, MOD5, MOD6 and MOD9 may be the $r_{2}, r_{5}, r_{6}$ and $r_{9}$ of Fig. 10, respectively that do not converge to the same angle. On the contrary, if it resides at a $2 \mathrm{~m}$ distance and the measured success rates are $\mathrm{r}_{2}, \mathrm{r}_{5}, \mathrm{r}_{6}{ }_{6}$ and $\mathrm{r}_{9}$, then all these success rates converge to the same angle $\left(35^{\circ}\right.$ or $\left.+35^{\circ}\right)$.

A second IRTX device is used to break the symmetry at the right and the left side of the reference infrared transmitter. Such curves can also be used to give an indication about the orientation of the target since the position estimation is expressed in polar coordinates (distance, angle) as described in [18].

The smooth success rate behavior designated by the curves of Figure 10 is valid only if the multiple IRTX devices do not transmit concurrently in order to avoid scrambling. This restriction slows down the localization procedure since the estimation time needed is longer in order to allow the target to receive the necessary pattern codes from all the neighboring IRTX devices.

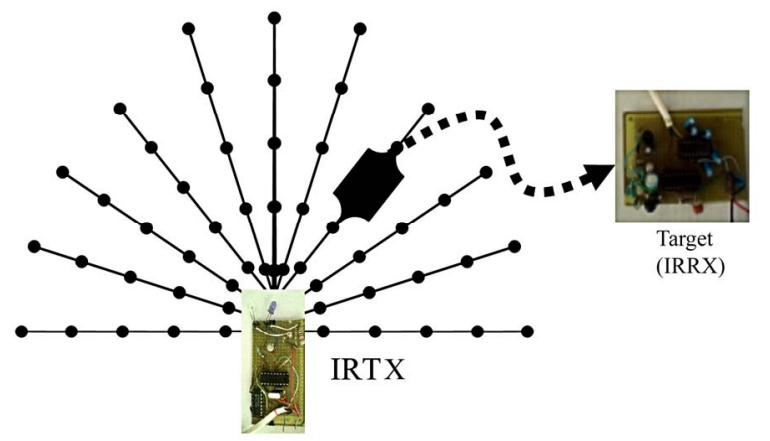

Figure 9. Topology for polar coordinate estimation

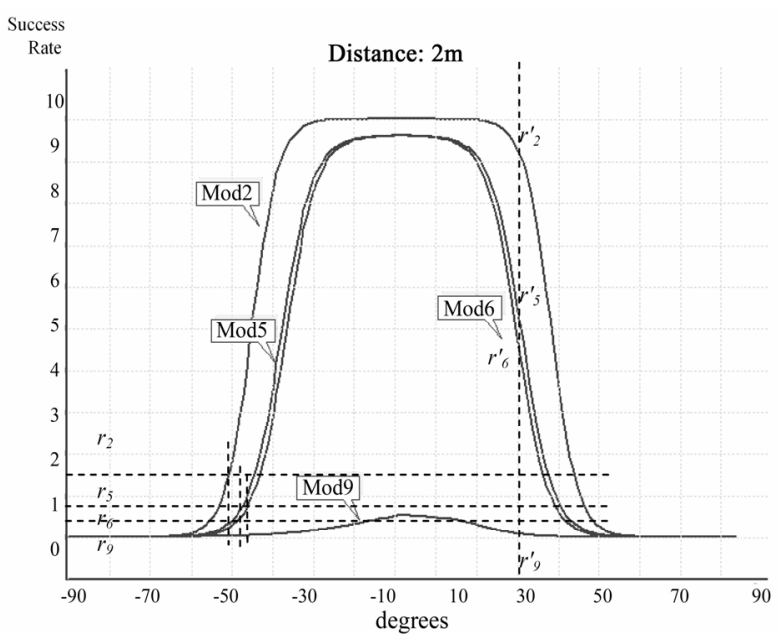

Figure 10. Success rates at $2 \mathrm{~m}$ distance from IRTX. 
The topology proposed at $[16,17]$ overcomes this restriction since the target moves with 2 degrees of freedom on a virtual grid plane as shown in Figure 11. The transmitters are positioned at the borders of the covered area. During the calibration stage, the target visits the grid nodes and stores the retrieved success rates. At real time operation, the closer grid node is selected after comparing the current success rates with the stored ones. An interpolation method can give a more accurate approximation of the real target position expressed in Cartesian coordinates. A larger area can be covered if more than two IRTX devices are properly positioned. An example of how the success rate of a specific pattern type changes within a $1 \mathrm{~m}^{2}$ area covered by 2 IRTX devices is shown in Figure 12. Comparing Figure 10 with Figure 12 we conclude that the success rates change in a more random way when the IRTX devices transmit concurrently.

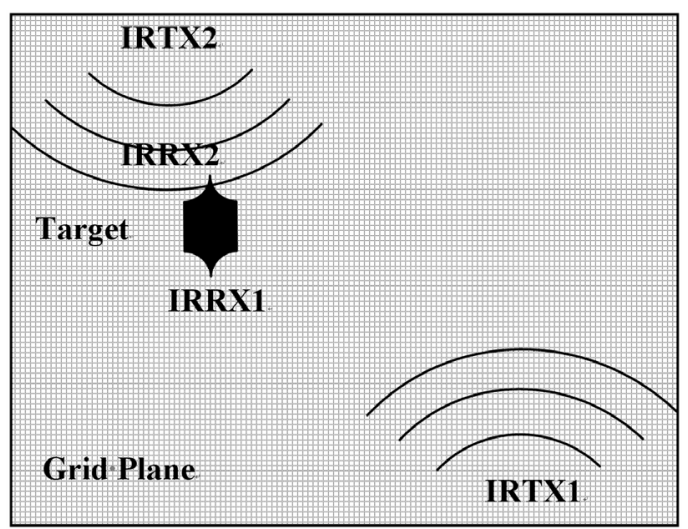

Figure 11. Grid topology.

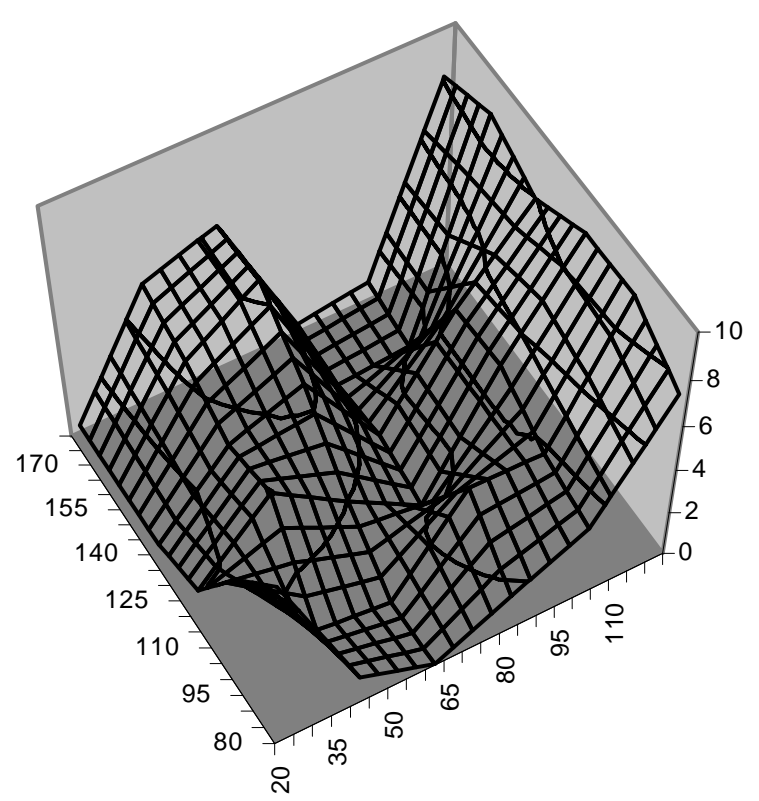

Figure 12. Example success rate in a $1 \mathrm{~m}^{2}$ area when the IRTX devices transmit concurrently.

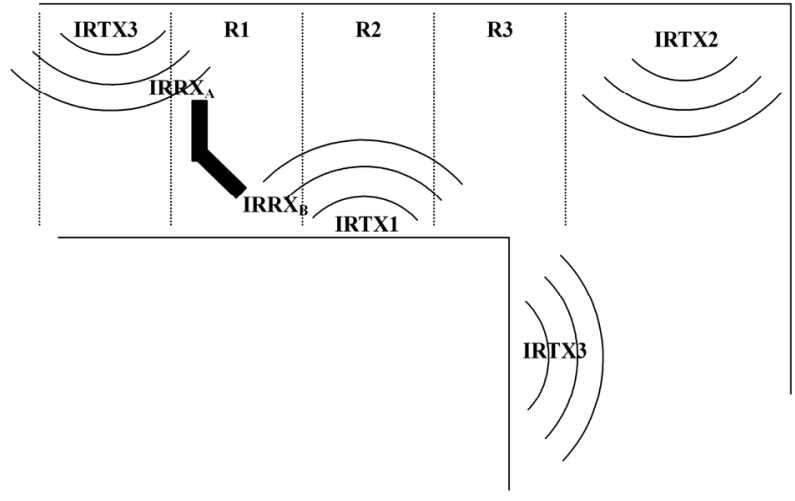

Figure 13. Example positioning of the IRTX devices in order to estimate both distance and orientation.



Figure 14. Distinct orientations

\section{Proposed Topology and Estimation Method of the Target Position and Orientation}

The detailed topology and position estimation method proposed in this paper for obtaining an orientation indication except from the Cartesian coordinates is presented in this section. The IRTX devices have the architecture shown in Figure 1 and transmit the stored, pre-encoded 160-bit signatures concurrently. They can be positioned as shown in Figure 13, in order to cover a corridor or a hall. Each IRTX device transmits a different signature. An IRTX device should transmit different signatures from its neighboring IRTX devices. Multiple IRTX devices can be used to cover the desired area transmitting alternatively only 3 types of signatures. For example, the IRTX3 device at the bottom of Figure 13 should be followed by IRTX1, IRTX2 etc in order to extend the area covered. A virtual grid is assumed to cover the area of this corridor or hall. The nodes of this grid are visited during the calibration phase by the target in order to measure the success rates at various orientations and familiarize with the area. 
The two IRRX sensors that reside on the target, form a $135^{\circ}$ angle in order to accept from a different angle the signatures sent by neighboring transmitters. This is useful in order to define unique orientation identities. The received signatures along with their parity bits are corrected by a Turbo decoder that is implemented in software at the Host computer at this prototype level. The algorithm employed by the Turbo decoder is the SumProduct. The system can distinguish the 8 draft orientations of the target that are shown in Figure 14.

An indication about the orientation of the target can be extracted if we assume that each IRTX device covers three adjunct regions in front of it and at its left and right side. A target residing at the region in front of this specific IRTX device may receive strong or weak signal from it and a weak signal from either of its neighboring IRTX devices. If the target resides at the left of this specific IRTX device it can receive strong or weak signals from it and its neighboring IRTX device at the left. If the target resides at the right of this specific IRTX device it can receive strong or weak signals from him and his neighboring IRTX device at the right.

In order to define when a signal will be characterized as strong or weak we should define the success rate in the present architecture. As already mentioned a 160-bit signature is transmitted by each IRTX device. This signature is accompanied by a 160-bit parity that has been generated after encoding the initial signature using the RSC encoder of Figure 3. The signature is interleaved by a random interleaver and encoded by the same RSC encoder. An IRTX device transmits the signature in its original and interleaved form as well as all the parity bits i.e., a total of 640 -bits are sent $(\mathrm{Rate}=1 / 4)$. The 480 redundant bits are used by the receiver in order to correct the burst errors that occurred during the transmission due to instant noise and recover the original 160-bit signature.

The original 160-bit signature is split in ten 16-bit parts consisting of different patterns of 1's and 0 's in order to differentiate the difficulty in correcting each one of these parts. For example, the correction of the signature parts 0x5555 and 0xEEEE may not have the same success. The number of signature part bits that match the expected ones after the Turbo Decoding, define the suc- cess rate (SR $i)$ for this signature part $i$. Its value may range from 0 to 16 . The success rate of the whole signature is a complex identity consisting of the 10 individual success rates SRi. The Averaged Success Rate (ASR) of a signature is defined as:

$$
A S R=\left(S R_{0}+S R_{1}+\ldots+S R_{9}\right) / 10
$$

The success rate of more than one sample can be averaged to get a more reliable estimation. An IRTX signal is characterized weak if the ASR of its signature is less or equal to 5 and strong if it is higher than 5 .

Focusing on the regions R1, R2 and R3 of Figure 13 and assuming that the target is at the specific position and has a North orientation we can state and experimentally prove that the $\operatorname{IRRX}_{\mathrm{A}}$ receives a weak signal from IRTX3 and IRRX $\mathrm{B}_{\mathrm{B}}$ receives a strong signal from IRTX1. In the same way the rules listed in Table 2 can be extracted for the topology of Figure 13. The columns A and $\mathrm{B}$ of this table designate the strength of the signals received from each IRTX device on the sensors IRRX and IRRX $_{B}$ respectively. The letters $\mathrm{W}$ and $\mathrm{S}$ mean Weak and Strong respectively, while the number following this letter corresponds to the IRTX device transmitting this signal. For example, W3 means that a weak signal is received from IRTX3.

The draft signal characterizations used in Table 2, form an orientation identity of the target. Hence, the target determines its orientation in a specific region as a first stage of the localization procedure. Unfortunately, the East orientation of R1 can be confused with the South East one of R2, as we can see from this table. Moreover, two target orientations of R2 and R3 (the ones with the shaded background) can also be confused. The higher number of orientations that can be potentially confused in this case is owed to the corner of the corridor and the IRTX2 and IRTX3 positioning. Such a problem can be resolved at a certain extent if we know some a priori restrictions (maximum/minimum speed, steering rules etc) in the move of the target as discussed in [16].

After determining the orientation of the target, a specific set of signature success rate maps like the one presented in Figure 12 are used to select the closer grid node to the target. More specifically, the success rates SR $i$

Table 2. Orientation rules.

\begin{tabular}{|c|c|c|c|c|c|c|c|c|}
\hline \multicolumn{3}{|c|}{ R1 } & \multicolumn{3}{c|}{ R2 } & \multicolumn{3}{c|}{ R3 } \\
\hline Orientation & A & B & Orientation & A & B & Orientation & A & B \\
\hline N & W3 & S1 & N & W2+W3 & W1 & N & S2 & W2 \\
\hline NE & W1+W3 & W1 & NE & W2 & S1 & NE & W2 & W3 \\
\hline E & W1 & W1+W3 & E & W2 & W1 & E & W2 & W1+W3 \\
\hline SE & S1 & W3 & SE & W1 & W1+W3 & SE & W2 & W1 \\
\hline S & W1 & S3 & S & S1 & W3 & S & W3 & W2 \\
\hline SW & W1+W3 & W3 & SW & W1 & W2+W3 & SW & W1+W3 & S2 \\
\hline W & W3 & W1+W3 & W & W1 & W2 & W & W2+W3 & W1 \\
\hline NW & S3 & W1 & NW & W3 & W1 & NW & W1+W2 & W2 \\
\hline
\end{tabular}


retrieved at the current position are compared to the corresponding stored ones from the calibration phase in order to select the closest grid node. The comparison is based on the following equation:

$$
D_{j}=\sum_{i=0}^{9} a b s\left(S R_{i}-S R_{i j}^{\prime}\right)
$$

The parameter $S R_{i}$ is the success rate of the signature part $i$ at the current target position. $S R_{i j}{ }^{\prime}$ is the success rate of the corresponding part $i$, retrieved during calibration at the node $j$ when the target had the orientation recognized at the first stage of the localization procedure. A different $D_{j}$ parameter is estimated for each grid node and the node that has the smallest $D_{j}$ value is selected as the closest one. This is the second stage of the localization procedure.

It should be emphasized that during the calibration phase, a different set of success rate maps should be stored for each orientation. For example, if a $1.8 \times 1.8$ meter region is assumed to be covered by a $30 \times 30 \mathrm{~cm}$ grid, then 25 grid nodes should be visited by the target without changing his orientation. A total of $8 \times 25=200$ success rate measurements should be carried out during the calibration phase to familiarize with a specific region, since 8 distinct orientations are considered.

A third localization procedure stage may follow, in which the actual target position can be further approximated by an interpolation method as described in [16]. This interpolation method assumes that the success rate parameters change linearly between neighboring grid nodes. This assumption is valid if the grid node distance is short enough (less than $30 \mathrm{~cm}$ ).

\section{Case Study-Discussion}

Considering the first stage of the localization procedure where the draft target orientation is decided, the successful estimations approach a level of $100 \%$. Some failures may occur at positions or orientations where the measured success rates are close to the limit between the Weak and Strong signal characterization. Of course, if the orientation estimation fails, the second and third stage of the localization procedure will possibly fail too, since a wrong success rate set of maps derived from the calibration stage will be used.
If the $1^{\text {st }}$ stage of the localization procedure is successful, then the expected absolute error at the position estimation that is carried out at the $2^{\text {nd }}$ and $3^{\text {rd }}$ localization stage should be comparable to the one achieved at [16]. The worst error in this case was equal to the grid node distance $(20 \mathrm{~cm})$. Having in mind that a longer grid node distance $(30 \mathrm{~cm})$ has been selected in the present work, the worst position estimation error is $30 \mathrm{~cm}$.

The worst error may be reduced if shorter grid node distance is selected. The $3^{\text {rd }}$ localization procedure stage (interpolation) would also be more efficient in this case since the success rate changes more linearly between neighboring nodes with $20 \mathrm{~cm}$ distance than those that have a $30 \mathrm{~cm}$ distance. Nevertheless, a time consuming calibration stage would be necessary since the number of nodes that have to be visited is extremely higher in this case. Moreover, if a large number of grid nodes are considered, there is a higher possibility that several nodes with similar success rate identities that can be confused at real time operation leading to false position estimations will be existed.

Similar problems need to be faced if more than 8 distinct target directions are considered. For example, if we try to distinguish between 16 directions the number of grid nodes that would have to be visited during calibration would be doubled. More than two signal characterizations would be required in this case to form different orientation identities (e.g., weak, medium, strong) but if a higher number of signal characterizations are used, then it is more difficult to distinguish the limits between them and more errors will appear when determining the target orientation.

The carrier frequency used at the prototype level is a low standard frequency of $38 \mathrm{KHz}$ that is adopted by commercial IR remote control devices. The estimation of the target orientation and position requires more than 1 sec in this case. This time can be reduced to less than $50 \mathrm{~ms}$ if a higher carrier frequency is used (e.g., $1 \mathrm{MHz}$ ) $[16,17]$. In this case, a custom bandpass filter and carrier rejecting circuit is required at the side of the receiver while the receiver processing unit should operate at higher clock frequencies (at least $20 \mathrm{MHz}$ ) to sample efficiently the IRRX sensors.

In Figure 15 an experimental target track in three regions (R1, R2, R3) neighboring to the IRTX1 transmitter

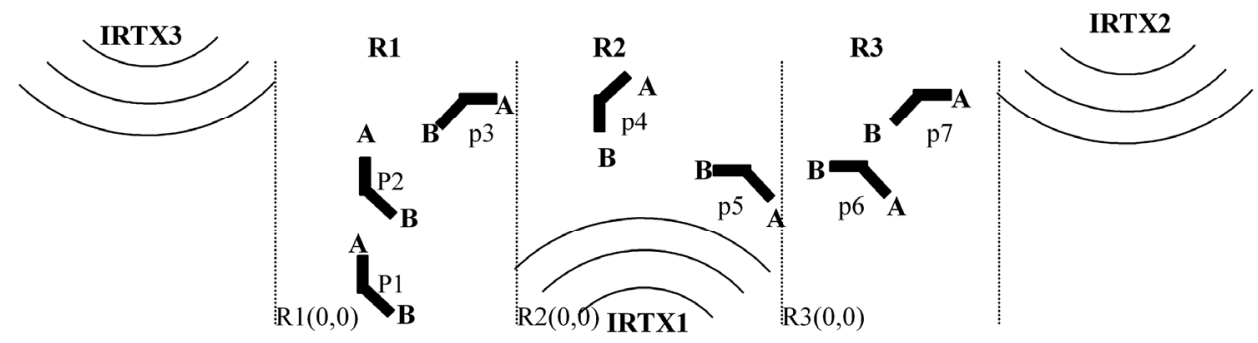

Figure 15. Experimental target track. 
Table 3. Experimental target localization results.

\begin{tabular}{|c|c|c|c|c|c|c|c|c|c|}
\hline \multirow{3}{*}{$\begin{array}{l}\text { Posit. } \\
\text { Ident. }\end{array}$} & \multicolumn{2}{|c|}{ Real Position } & \multicolumn{4}{|c|}{ Experimentally Estimated Position } & \multirow{3}{*}{$\begin{array}{c}\operatorname{IRRX}_{\mathbf{A}} \\
\text { ASR }\end{array}$} & \multirow{3}{*}{$\begin{array}{c}\text { IRRX }_{\text {B }} \\
\text { ASR }\end{array}$} & \multirow{3}{*}{$\begin{array}{c}\text { FEC } \\
\text { Used/ } \\
\text { Inter } \\
\text { pol. }\end{array}$} \\
\hline & \multirow{2}{*}{ Coordinates } & \multirow{2}{*}{ Orient } & \multicolumn{2}{|c|}{ Coordinates } & \multicolumn{2}{|c|}{ Orientation } & & & \\
\hline & & & Coord. & Stab & Orient. & Stab & & & \\
\hline $\mathrm{p} 1$ & $\begin{array}{c}\mathrm{R} 1(80 \mathrm{~cm}, \\
30 \mathrm{~cm})\end{array}$ & $\mathrm{N}$ & $\begin{array}{c}\mathrm{R} 1(60 \mathrm{~cm} \\
0)\end{array}$ & $1 / 3$ & $\mathrm{~N}$ & $3 / 3$ & 2(3) & $6(1)$ & $\begin{array}{l}\mathrm{Y} \\
/ \mathrm{N}\end{array}$ \\
\hline $\mathrm{p} 2$ & $\begin{array}{c}\mathrm{R} 1(80 \mathrm{~cm}, \\
90 \mathrm{~cm})\end{array}$ & $\mathrm{N}$ & $\begin{array}{c}\mathrm{R} 1(90 \mathrm{~cm} \\
90 \mathrm{~cm})\end{array}$ & $2 / 3$ & $\mathrm{~N}$ & $3 / 3$ & $3(3)$ & $10(1)$ & $\begin{array}{l}\mathrm{N} \\
/ \mathrm{N}\end{array}$ \\
\hline p3 & $\begin{array}{c}\mathrm{R} 1(140 \mathrm{~cm} \\
160 \mathrm{~cm})\end{array}$ & $\mathrm{E}$ & $\begin{array}{c}\mathrm{R} 1(150 \mathrm{~cm} \\
180 \mathrm{~cm})\end{array}$ & $2 / 3$ & E & $3 / 3$ & $1(1)$ & $\begin{array}{l}1(1) \\
1(3) \\
\end{array}$ & $\begin{array}{l}\mathrm{Y} \\
/ \mathrm{N} \\
\end{array}$ \\
\hline $\mathrm{p} 4$ & $\begin{array}{c}\mathrm{R} 2(70 \mathrm{~cm}, \\
160 \mathrm{~cm})\end{array}$ & NE & $\begin{array}{c}\mathrm{R} 2(50 \mathrm{~cm} \\
150 \mathrm{~cm})\end{array}$ & $3 / 3$ & NE & $3 / 3$ & $1(2)$ & $7(1)$ & $\begin{array}{l}\mathrm{N} \\
/ \mathrm{Y}\end{array}$ \\
\hline p5 & $\begin{array}{c}R 2(160 \mathrm{~cm}, \\
80 \mathrm{~cm})\end{array}$ & $S E$ & $\begin{array}{c}R 2(120 \mathrm{~cm}, \\
90 \mathrm{~cm})\end{array}$ & $1 / 3$ & $E$ & $2 / 3$ & $\begin{array}{l}2(1) \\
1(2)\end{array}$ & 2(1) & $\begin{array}{l}Y \\
/ N \\
\end{array}$ \\
\hline p6 & $\begin{array}{c}\mathrm{R} 3(50 \mathrm{~cm}, \\
80 \mathrm{~cm})\end{array}$ & SE & $\begin{array}{c}\mathrm{R} 3(30 \mathrm{~cm} \\
60 \mathrm{~cm})\end{array}$ & $2 / 3$ & SE & $2 / 3$ & $\begin{array}{l}1(1) \\
2(2)\end{array}$ & $3(1)$ & $\begin{array}{l}\mathrm{Y} \\
/ \mathrm{N} \\
\end{array}$ \\
\hline p7 & $\begin{array}{c}\mathrm{R} 3(100 \mathrm{~cm} \\
170 \mathrm{~cm})\end{array}$ & E & $\begin{array}{c}\mathrm{R} 3(120 \mathrm{~cm} \\
150 \mathrm{~cm})\end{array}$ & $1 / 3$ & E & $3 / 3$ & $4(2)$ & $5(1)$ & $\begin{array}{l}\mathrm{Y} \\
/ \mathrm{N}\end{array}$ \\
\hline
\end{tabular}

Table 4. Localization method comparison.

\begin{tabular}{|c|c|c|c|}
\hline Method & Complexity/Cost & Range & $\begin{array}{c}\text { Accuracy/ } \\
\text { Orientation Indication }\end{array}$ \\
\hline This work & Very Low & $16 \mathrm{~m}^{2} /$ transmitter & $\begin{array}{c}20-30 \mathrm{~cm} / \\
\text { Yes }(<45 \text { degrees })\end{array}$ \\
\hline $\begin{array}{l}\text { Acoustic sound (time of flight/ } \\
\text { reception angle) }\end{array}$ & Medium & Tens of meters & $\begin{array}{c}10 \mathrm{~cm} / \\
\text { Yes (5 degrees) }\end{array}$ \\
\hline $\begin{array}{c}\text { Ultrasonic } \\
\text { (time of flight) }\end{array}$ & Medium & Tens of meters & $\begin{array}{l}2-3 \mathrm{~cm} / \\
\text { No }\end{array}$ \\
\hline $\begin{array}{c}\text { Ultrasonic } \\
\text { (phase difference) }\end{array}$ & High & $10-20 \mathrm{~m}^{2}$ & $\begin{array}{l}\text { Few centimeters/ } \\
\text { Yes }(<20 \text { degrees })\end{array}$ \\
\hline $\begin{array}{c}\text { Laser } \\
\text { (time of flight) }\end{array}$ & High & One hundred meters or longer & $\begin{array}{c}2-3 \mathrm{~cm} / \\
\text { Indirectly }\end{array}$ \\
\hline Image Processing & Very High & Tens of meters & $\begin{array}{c}\text { Few centimeters / } \\
\text { Indirectly }\end{array}$ \\
\hline $\begin{array}{l}\mathrm{RF} \text { (Received Signal Strength } \\
\text { Indicator) }\end{array}$ & Medium & $2-3 m$ & $\begin{array}{l}\text { Tens of centimeters/ } \\
\text { No }\end{array}$ \\
\hline
\end{tabular}

is presented. Each one of these regions has an area of $1.8 \mathrm{mX} 1.8 \mathrm{~m}$ and is covered by a virtual grid with $30 \mathrm{~cm}$ node distance. It should be noted that a single IRTX device can cover an area of up to $16 \mathrm{~m}^{2}$ but the areas covered by two neighboring IRTX devices should overlap in order to define rules like the ones presented in Table 2. It will also be assumed that the lower left corner of a region Ri has coordinates $R_{i}(0,0)$ as shown in Figure 15 .

Table 3 lists the real target coordinates and orientations and the experimentally measured ones. Three samples have been examined for every position and the experimental coordinates and orientation listed in Table 3 are the best ones achieved from these 3 samples. The $5^{\text {th }}$ and $7^{\text {th }}$ columns are an indication of the results' stability denoted as $\mathrm{x} / 3$ where $\mathrm{x}$ shows how many samples among the 3 considered leaded to the selected best results. In the ASR columns the Average Success Rates measured by IRTX $_{\mathrm{A}}$ and IRTX $\mathrm{B}_{\mathrm{B}}$ are listed and these values were used for the estimation of the best position/ orientation mentioned in this table. The number in the parentheses in these two columns refers to the IRTX device that corresponds to the listed ASR. The last column shows the positions where the Forward Error Correcting method (FEC) and the $3^{\text {rd }}$ stage of the localization procedure improved the results.

It is worth mentioning the case of p5 where the system was unable to reach the right decision about the orientation of the target (decided $\mathrm{E}$ instead of SE). Consequently, the estimated coordinates could not be close enough to the real target position. The signal characterization extracted at $\mathrm{p} 5$ did not match any orientation and the conclusion for the E orientation was reached by ignoring the Weak indication from IRTX1.

The stability of the orientation results is also very good since in most positions the right orientation was constantly selected. The stability of the position coordinates is slightly worse, but rules like the ones described in [16] that were not used in the present case study can assist the system to improve its results. In many cases, the Turbo Decoding method led to better results by discarding instant noise effects as indicated by the last column of Table 3 . Finally, the $3^{\text {rd }}$ localization stage was helpful only once. This is due to the fact that the grid node distance used was relatively long. 
Table 4 provides a general comparison between various localization methods. As already mentioned the cost of the proposed solution is extremely low since it is sufficient to count the received patterns by incorporating slow-speed microcontrollers without the necessity to employ high-precision sensors. Moreover, a large area can be covered by a small number of infrared transmitters since a single transmitter can cover a $16 \mathrm{~m}^{2}$ area. The accuracy in the measured distance or the orientation is quite good for several applications. Acoustic sound and ultrasonic waves can achieve a better accuracy and they can also provide some orientation indication but they require significantly higher cost sensors and processing units in order to discriminate small time intervals or phase shifts. Laser beams can achieve a very good accuracy and can cover a wide range but they have significantly higher cost since they require much faster processing units. They also provide orientation indication in an indirect manner since they are used to measure a distance at a specific direction. Image processing also requires very high cost complicated sensors and processing units in order to analyze a captured image. Orientation can be concluded indirectly if the target is aware of his environment by detecting specific landmarks in his direction. Finally, Received Signal Strength Indicator (RSSI) can also provide a draft distance estimation of the $\mathrm{RF}$ receiver.

\section{Conclusions}

A localization system capable of determining the orientation of a target in one of 8 possible directions with high estimation stability was described in this paper. Beside this draft orientation, the system is also capable to determine the position of the target with a maximum absolute error less than $30 \mathrm{~cm}$. The estimation method is based on a simple enumeration of infrared patterns in order to estimate a success rate parameter that qualifies the signal of a specific transmitter. Each infrared transmitter covers an area of up to $16 \mathrm{~m}^{2}$ and multiple transmitters with partially overlapping range can be used to cover the desired area. The infrared patterns are received by two sensors that are positioned at an angle of $135^{\circ}$ on the target in order to differentiate the quality of reception from different transmitters. Simple commercial components have been used at the side of the transmitter and the receiver, since no high precision analog measurements are required, leading to ultra low cost implementations.

Future work will focus on increasing the distinct directions that can be distinguished at the various target positions without sacrificing the accuracy, stability and speed of the estimation method. The localization of a target in 3 dimensions with 4 degrees of freedom (including rotation) using a similar method than the one presented here will also be investigated.

\section{References}

[1] A. Clerentin, A. Delahoche, E. Brassart and C. Drocourt, "Self localisation: A new uncertainty propagation architectur," Elsevier Robotics and Autonomous Systems, Vol. 51, pp. 151-166, 2005.

[2] J. Borenstein, B. Everett and L. Feng, "Navigating mobile robots: Systems and techniques," A.K. Peters Ltd Wellesley, 1996.

[3] J. Miura, Y. Negishi, and Y. Shirai, "Adaptive robot speed control by considering map and motion uncertainty," Elsevier Robotics and Autonomous Systems, Vol. 54, pp. 110-117, 2006.

[4] P. Smith and K. Zografos, "Sonar for recognizing the texture of pathways," Robotics and Autonomous Systems, Vol. 51, pp. 17-28, 2005.

[5] M. Minami, Y. Fukuju, K. Hirasawa, S. Yokoyama, M. Mizumachi, H. Morikawa, and T. Aoyama, "Dolphin: A practical approach for implementing a fully distributed indoor ultrasonic positioning system," Lecture Notes for Computer Science (LNCS), Vol. 3205, pp. 347-365, 2004.

[6] A. Ladd, K. Bekiris, A. Rudys, L. Kavraki, and D. Wallach, "Robotics based location sensing using wireless ethernet," Wireless Networks, Vol. 11, No. 1-2, pp. 189-204, January 2005.

[7] C. Flora, M. Ficco, S. Russo, and V. Vecchio, "Indoor and outdoor location based services for portable wireless devices," Proceedings of 1st IEEE International Workshop on Services and Infrastructure for Ubiquitous and Mobile Internet, Columbus OH, pp. 244-250, June 2005.

[8] J. Kosel, H. Pfutzner, L. Mehnen, E. Kaniusas, T. Meydan, N. Vazquez, M. Rohn, A. Merlo, and B. Marquardt, "Non contact detection of magnetoelastic position sensors," Elsevier Sensors and Actuators A, No. 123-124, pp. 349-353, 2005.

[9] V. Schlageter, P. Besse, R. Popovic, and P. Kucera, "Tracking system with $5 \mathrm{deg}$ of freedom using a $2 \mathrm{~d}$ array of hall sensors and a permanent magnet," Elsevier Sensors and Actuators A, No. 92, pp. 37-42, 2001.

[10] E. Prigge and J. How, "Signal architecture for distributed magnetic local positioning system," IEEE Sensors Journal, Vol. 4, No. 6, pp. 864-873, 2004.

[11] T. Jin, J. Lee, and S. Tso, “A new space and time sensor fusion method for mobile robot navigation," Wiley Journal of Robotics Systems, Vol. 21, No. 7, pp. 389-400, 2004.

[12] E. Bicho, P. Mallet, and G. Schoner, "Target representation on an autonomous vehicle with low level sensors," International Journal of Robotics Research, Vol. 19, No. 5, pp. 424-447, May 2000.

[13] T. Aytac and B. Barshan, "Simultaneous extraction of geometry and surface properties of targets using simple infrared sensors," SPIE Optical Engineering Journal, Vol. 43, No. 10, pp. 2437-2447, October 2004.

[14] G. Benet, F. Blanes, J. Simo, and P. Perez, "Using infrared sensors for distance measurement in mobile robots," 
Robot Autonomy Systems, Vol. 30, pp. 255-266, 2002.

[15] N. Petrellis, N. Konofaos, and G. Ph. Alexiou, "Target localisation utilising the success rate in infrared pattern recognition," IEEE Sensors Journal, Vol. 6, pp. 13551364, 2006.

[16] N. Petrellis, N. Konofaos, and G. Ph. Alexiou, "Using future position restriction rules for stabilizing the results of a noise sensitive indoor localization system," SPIE Optical Engineering Journal, Vol. 6, No. 46, Article No 067202, pp. 067202-1-067202-11, 2007.

[17] N. Petrellis, F. Gioulekas, M. Birbas, J. Kikidis, and A. Birbas, "Use of interleaving and error correction to infrared patterns for the improvement of position estimation systems," in Proceedings of IEEE Conference Emerging Technologies-Factory Automation, Hamburg, Germany, pp. 888-891, September 2008.

[18] N. Petrellis, N. Konofaos, and G. Ph. Alexiou, "A wireless infrared sensor network for the estimation of the position and orientation of a moving target," in Proceedings
ICST-ACM Conference MobiMedia'07, Nafpaktos, Greece, August 2007.

[19] M. Arzel, C. Lahuec, F. Seguin, D. Gnaedig, and M. Jezequel, "Semi-iterative analog turbo decoding," IEEE Transactions on Circuits and Systems, Vol. 54, No. 6, pp. 1305-1315, 2007.

[20] C. Schlegel and L. Perez, "Trellis and turbo coding," IEEE Series on Digital \& Mobile Communication, Wiley Interscience, 2004.

[21] F. Gioulekas, M. Birbas, A. Birbas, and G. Bilionis, "Analog error-correcting decoders using SiGe BiCMOS technology," International Journal of Analog Integrated Circuits and Signal Processing, Springer, Vol. 52, No. 3 pp. 117-132, October 2007.

[22] M. A. Bickerstaff, D. Garret, T. Prokop, C. Thomas, and C. Nicol, "A $24 \mathrm{Mb} / \mathrm{s}$ radix-4 logMAP turbo decoder for 3GPP-HSDPA mobile wireless," in Proceedings IEEE Conference International Solid-State Circuits, San Francisco, CA, pp. 150-151, February 2003. 